First publ. in: Journal of Low Temperature Physics 101 (1995), 1-2, pp. 211-216

\title{
Quantum fluids on alkali metal surfaces
}

\author{
G.Mistura, D.Reinelt, S.Herminghaus, P.Leiderer and \\ M.H.W.Chan*
}

Fakultät für Physik, Universität Konstanz, Postfach 5560, 78434 Konstanz, Germany

*Dept. of Physics, Pennsylvania State University, University Park, PA 16802, USA

The behaviour of quantum fluid films on alkali metal surfaces is investigated. Using the quartz microbalance technique, the wetting phase diagram can be explored by adsorption isotherm measurements. Optically excited surface plasmons are used to obtain real-time images of the dynamical behaviour of ${ }^{4} \mathrm{He}$ droplets on cesiated surfaces.

PACS numbers: $05.70 \mathrm{Ln}, 05.70 \mathrm{Jk}, 64$.

\section{INTRODUCTION}

After the prediction that ${ }^{4} \mathrm{He}$ does not wet the heavy alkali metal surfaces, ${ }^{1}$ a vigorous experimental research effort was started. By now, it has been firmly established that ${ }^{4} \mathrm{He}$ adsorbed on Cs undergoes a wetting transition around $2 \mathrm{~K},{ }^{2}$ while the case of ${ }^{4} \mathrm{He}$ on $\mathrm{Rb}$ is still not completely clear. Quartz microbalance data ${ }^{3}$ indicate that the wetting temperature $T_{w}$ of ${ }^{4} \mathrm{He}$ on $\mathrm{Rb}$ is below $1.6 \mathrm{~K}$, the lowest temperature studied by the Penn State University group. Heat transport measurements suggest that $T_{w}=0$ $\mathrm{K} .{ }^{4}$ More recently, a low temperature mass flow study of ${ }^{4} \mathrm{He}$ across a $\mathrm{Rb}$ ring finds a $T_{w}=0.3 \mathrm{~K} .{ }^{5}$ However, the slope of the prewetting line determined by the Exeter group is about $10^{3}$ times larger than the theoretical estimate. ${ }^{5}$

Although most of the experimental investigations have been devoted to ${ }^{4} \mathrm{He}, \mathrm{H}_{2}$ too has been predicted to undergo a wetting transition along the liquid-vapor coexistence line when adsorbed on heavy alkali metal surfaces. ${ }^{6}$ On Rb such a transition has been observed at $T_{w}=19 \mathrm{~K}^{7}$ Most of this 
research has been carried out with a quartz microbalance. As it will be described in the following paragraph, this is a powerful technique that accurately determines the growth of an adsorbed film. However, it does not provide any information about the dynamics of the prewetting transition, nor about the film profile. These problems can be more appropriately addressed using optically excited surface plasmons. This paper is divided in two parts. In the first one we will briefly describe the quartz microbalance technique, together with its advantages and its limitations for the study of physisorption on alkali metal surfaces. To better illustrate these points, the complete prewetting phase diagram of $\mathrm{H}_{2}$ on $\mathrm{Rb}$ with such a technique will be shown. In the second part, we will explain the experimental set-up used to study surface plasmons excited at the interface between an alkali metal surface and an adsorbed film and we will present some preliminary results showing the behavior of ${ }^{4} \mathrm{He}$ droplets on a Cs surface.

\section{THE QUARTZ MICROBALANCE}

The heart of our quartz microbalance is an AT-cut quartz crystal. It is a thin quartz disk, with a gold electrode, evaporated at the center of each crystal face. When a voltage is applied to the two gold electrodes at a frequency very close to the crystal's shear resonance, the two faces move in a shear mode. If a small mass $\mathrm{dm}$ is deposited onto each gold electrode, the quartz resonance frequency drops by an amount proportional to $\mathrm{dm} .{ }^{8} \mathrm{In}$ our case, the adsorption of a solid-like layer of $\mathrm{H}_{2}$ corresponds to $0.67 \mathrm{~Hz}$ out of a resonance frequency near $16.6 \mathrm{MHz}$. The frequency resolution of our microbalance is of about $0.03 \mathrm{~Hz}$. Facing the two gold electrodes there are two alkali metal dispensers. A current is passed through them to evaporate the alkali metal. This process is performed at low temperature and with a shutter to reduce the risk of chemical contamination of the surface. We do not have any direct way to determine how smooth and pure the alkali metal film is. All of our information on the surface quality is drawn from adsorption isotherms. An adsorption isotherm is determined by measuring, at a constant temperature, how the quartz resonance frequency varies with the pressure $P$ of the vapor in equilibrium with the adsorbed film.

Figure 1a shows the final portions of a set of adsorption isotherms of $\mathrm{H}_{2}$ on Rb. On this expanded scale the isotherm at $18.89 \mathrm{~K}$ looks flat, indicating that at this high temperature, well above its bulk triple point of $13.80 \mathrm{~K}$, $\mathrm{H}_{2}$ does not wet $\mathrm{Rb}$. If the temperature is increased to $19.43 \mathrm{~K}$ a sharp drop in frequency is observed very close to the saturated vapor pressure, $\mathrm{P}_{0}$. As $\mathrm{T}$ is further increased, this step moves to lower relative pressures, becomes smaller and, at higher temperatures, more rounded. These sharp steps re- 

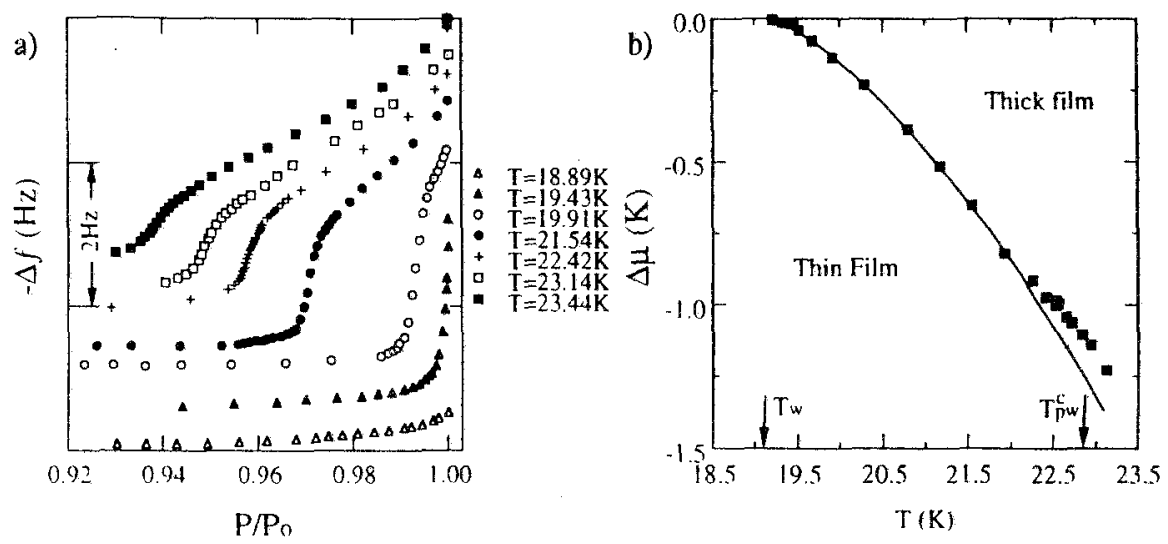

Fig. 1. a) Adsorption isotherms of $\mathrm{H}_{2}$ on $\mathrm{Rb}$ taken across the wetting temperature $T_{w}=19.10 \mathrm{~K} . \mathrm{b}$ ) Prewetting phase diagram of $\mathrm{H}_{2}$ on Rb.

present the prewetting or thin to thick film transition. Figure $1 \mathrm{~b}$ displays the location of the prewetting steps, expressed in terms of chemical potential difference relative to the saturation value, at various temperatures. We identify the position of each prewetting jump, $\mathrm{P}_{\text {step }} / \mathrm{P}_{0}$, as that corresponding to the maximum slope of the isotherm. Near $T_{w}$ the predicted equation of the prewetting line is $-\Delta \mu=a\left(T-T_{w}\right)^{3 / 2}$, with $a=0.188 K^{-1 / 2}$ for the $\mathrm{H}_{2} / \mathrm{Rb}$ system. ${ }^{7}$ The solid line in Fig. $1 \mathrm{~b}$ represents a nonlinear least squares fit of the data with $T \leq 22 \mathrm{~K}$ with the power law expressed above where $a, T_{w}$ and the exponent $\beta$ are parameters of the fit. The results of the fit are $T_{w}=19.10 \pm 0.06 \mathrm{~K}$, in excellent agreement with the predicted value of $18-22 \mathrm{~K}^{6} \beta=1.48 \pm 0.06$ and $a=0.176 \pm 0.016 \mathrm{~K}^{1-\beta}$.

These and other results ${ }^{2,6-8}$ clearly show that quantitative conclusions concerning the complete wetting phase diagram of an adsorbed film can be deduced with the quartz microbalance technique. Furthermore, the application of such a technique to the study of wetting on weak-binding surfaces is particularly facilitated by the planar geometry of the quartz crystal which makes it easy to evaporate an alkali metal onto it. On the other hand, in order to have a more complete understanding of the wetting transition which, as seen before, is finally accessible to experiments, complementary techniques are necessary.

\section{SURFACE PLASMON MICROSCOPY}

Surface plasmons (SP) are electromagnetic waves travelling on metal surfaces. Their typical frequencies are in the visible range of the spectrum 

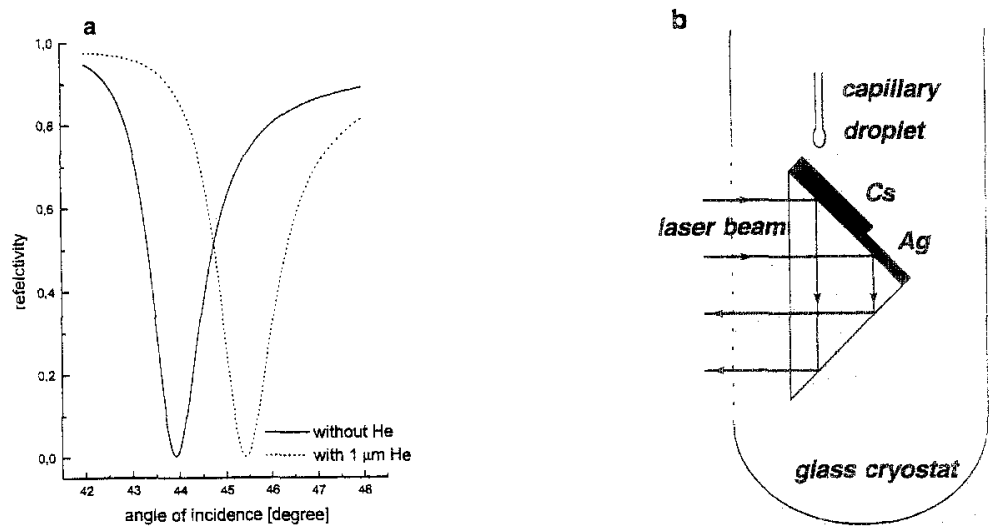

Fig. 2. a) Surface plasmon resonance curve calculated for a silver film with cesium overlayer. Dashed line: bare surface. Solid line: covered with $1 \mu \mathrm{m}$ of ${ }^{4} \mathrm{He}$. b) Experimental setup. The prism is illuminated through the walls of the glass cryostat and serves for both excitation of the surface plasmons and retroreflection out of the cryostat.

which makes them well suited for use as an optical probe of processes taking place in close vicinity of the surface ${ }^{10}$ Light can be coupled to the SP by means of an evanescent wave, as for instance by illuminating the base of a prism under total internal reflection conditions (cf. Fig.2). When the metal whose surface is to be investigated is evaporated as a film of appropriate thickness (several hundred Ångstroms) onto the prism base, SP on the metal/vacuum interface are resonantly excited at a particular angle of incidence at expense of the reflected light. The presence of a thin film of, say, superfluid helium on the metal surface detunes the SP resonance as shown in Fig.2a. This allows for such a film to be detected with an accuracy of better than an Angstrom if a noble metal is used and if the resonance angle is measured with high resolution. ${ }^{11}$ If other metals are used, the sensitivity is somewhat inferior due to higher surface plasmon damping. When the sample is illuminated with an expanded laser beam and the reflected light is imaged onto a CCD camera chip, a so-called SP microscope results. Although the film thickness resolution is not as good as in high resolution setups, helium films with a few Angstroms thickness can be imaged with reasonable contrast and a temporal resolution limited only by the recording equipment. ${ }^{12,13}$

In our experiment, standard video equipment was used to record the dynamics of superfluid ${ }^{4} \mathrm{He}$ droplets falling onto a tilted cesiated surface. In Fig.2b, we depicted our sample cell which contains the prism (covered with a $330 \mathrm{~nm}$ silver film before the experiment), a capillary above the prism 


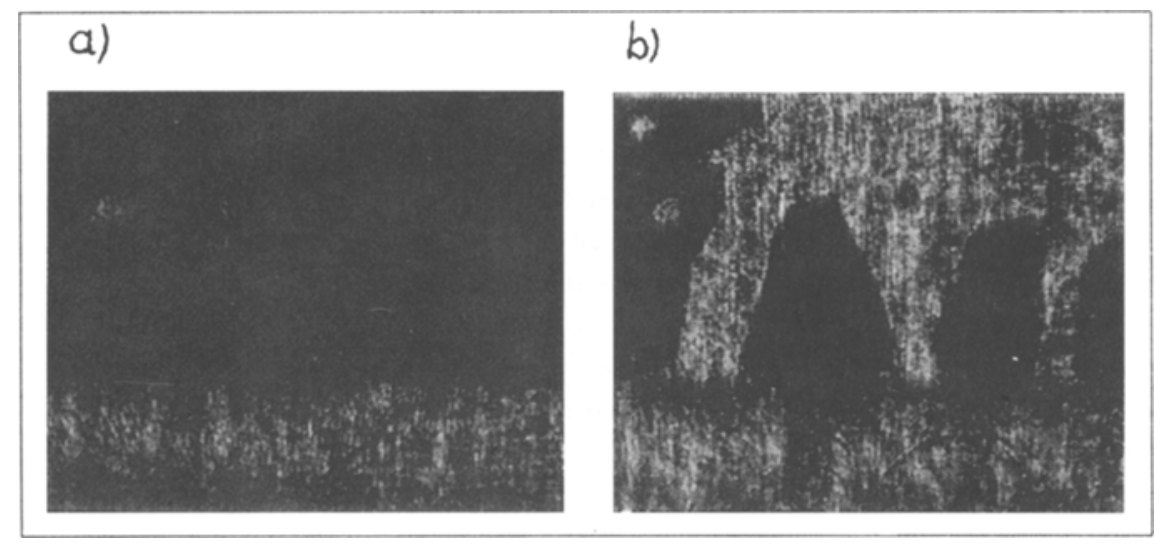

Fig. 3. a) Surface plasmon image of the bare sample. Dark: cesiated region (in resonance). Bright: non-cesiated (off resonance). The width of the pictures corresponds to $6 \mathrm{~mm}$. The is yet limited by the poor optical quality of of the glass cryostat walls. b) Immediately after a falling drop of ${ }^{4} \mathrm{He}$ came into contact $(\mathrm{T}=1.5 \mathrm{~K})$.

for supplying the liquid helium and a Cs dispenser. After cooling to $4.2 \mathrm{~K}$, the dispenser is heated and $\mathrm{Cs}$ is deposited onto the upper prism face. A shutter has been placed in front of the lower part of this prism face in order to provide a non-cesiated silver surface for comparison. This can be seen in Fig.3a which shows an image of the upper prism face, with the angle of incidence adjusted so as to match the SP resonance on the cesiated part. The latter thus appears dark, while the silver part, which is not in resonance, appears bright.

When a ${ }^{4} \mathrm{He}$ droplet falls down onto this surface, the SP in the region covered with helium will be detuned resulting in a substantial increase in brightness. This is shown in Fig.3b, which shows the surface just after a droplet has arrived at contact. Finger-like structures are clearly seen to evolve, indicating that the surface is not completely wet by the helium. It is observed that the helium flows down only along the finger-shaped channel regions generated immediately after contact with the substrate. No tendency of spreading is apparent. However, as soon as the helium reaches the silver surface, the liquid flow, which had been restricted to 'channels' on the cesiated part of the sample, strongly spreads laterally over the whole silver surface. This can be seen from the fact that there is a change in intensity virtually all over the silvered part, with some lateral structure indicating that the film thickness is not homogeneous.

It should be noted that a full contrast (dark-bright) change on the ce- 
siated part is achieved by coverage with approximately $1 \mu \mathrm{m}$ of helium. On the trailing edges of the fingers on the Cs (when the flow-down process of the drop was almost complete), we observed a quite gradual change in intensity over approximately one millimeter, indicating that what is flowing are not bulk fluid structures but rather thin helium films. From the wetted area of several square millimeters and a typical droplet volume of a few hundred picoliters, we can estimate the film thickness to be a few microns. In this case, one expects a critical flow velocity for vortex formation on the order of a few $\mathrm{cm} / \mathrm{sec} .{ }^{14}$ In fact, analyzing a series of pictures, we found the flow velocity to be approximately $5 \mathrm{~cm} / \mathrm{sec}$ which suggests that the film flow by which the drop drains down the surface is limited by the critical flow velocity.

\section{ACKNOWLEDGMENTS}

This research was partially supported by NSF grant No. DMR-90 22681 and by the Deutsche Forschungsgemeinschaft, SFB 306.

\section{REFERENCES}

1. E. Cheng, M.W. Cole, W.F. Saam and J. Treiner, Phys. Rev. Lett. 67, 1007 (1991).

2. See e.g. J.E. Rutledge and P. Taborek, Phys. Rev. Lett. 71, 263 (1993) and references therein.

3. G. Mistura, H.C. Lee and M.H.W. Chan, Physica 194-196B, 661 (1994).

4. N. Bigelow, P.J. Nacher and J. Dupont-Roc, J. Low Temp. Phys. 89, 135 (1992); B. Demolder, N. Bigelow, P.J. Nacher and J. Dupont-Roc, J. Low Temp. Phys. 98, 91 (1995).

5. A.F.G. Wyatt, J. Klier and P. Stefanyi, Phys. Rev. Lett. 74,1151 (1995).

6. E.Cheng, G. Mistura, H.C. Lee, M.H.W. Chan, M.W. Cole, C. Carraro, W.F.Saam and F. Toigo, Phys. Rev. Lett. 70, 1854 (1993).

7. G. Mistura, H.C. Lee and M.H.W. Chan, J. Low Temp. Phys. 96, 489 (1994).

8. M. Lea, P. Fozooni and P.W. Retz, J. Low Temp. Phys. 54, 303 (1984)

9. K. Ketola and R.B. Hallock, Phys. Rev. Lett. 71, 3295 (1993) and references therein.

10. J.R. Sambles, G.W: Bradberry, F. Yang, Contemp.Phys. 32, 173 (1991).

11. C.F. Eagen, W.H. Weber, Phys. Rev. B19, 5068 (1979).

12. B. Rothenhäusler, J. Rabe, P. Korpiun, W. Knoll, Surf.Sci. 137, 373 (1984).

13. S. Herminghaus, C. Bechinger, W. Petersen, P. Leiderer, Opt.Commun. 112, 16 (1994).

14. J. Wilks, 'The Properties of Liquid and Solid helium', Oxford, 1967. 